\title{
Searching for Be star candidates within large databases
}

\author{
Beatriz Sabogal ${ }^{1, \star}$ and Alejandro García-Varela ${ }^{1, \star \star}$ \\ ${ }^{1}$ Universidad de los Andes, Departamento de Física, Cra.1 No.18A-10, Ed. Ip, A.A.4976, Bogotá, Colombia
}

\begin{abstract}
Long time surveys yield many light curves among their byproducts, useful to identify candidate Be stars that can be confirmed by spectroscopic follow-ups. We present in this work different techniques we have used to find Be star candidates in the Galaxy, the Magellanic Clouds and the OGLE-IV Gaia South Ecliptic Pole Field.
\end{abstract}

\section{Introduction}

Be stars are non-supergiant very rapid rotators, with spectral types between late $\mathrm{O}$ and early $\mathrm{A}$, whose spectra show or have shown at some time one or more Balmer lines in emission ([1]). The photometric variations shown by these stars can exist in different time scales depending on their causes ([5]). Photometric searches for Be star candidates (BeSC hereafter) are useful in order to have larger samples of Be stars to analyze their dependency on metallicity and evolutionary state. A photometric search for BeSC within the Small Magellanic Cloud (SMC) OGLE-II catalog of variable stars was performed by [3]. Those authors found that, contrary to the Galactic Be stars, many light curves of BeSC had jumps of brightness and quasi-periodic variations.

\section{Techniques used in Searches for BeSC}

After the work of [3] we searched for BeSC within the Large Magellanic Cloud (LMC) OGLE-II catalog of variable stars, using photometric criteria. We looked for stars in the range of magnitudes, colors and amplitudes of Galactic Be stars. From a list of about 5000 stars, we selected 2446 BeSC that were visually inspected. Comparing these stars with those BeSC of the SMC, we found an important dependency of the amplitude and duration of outbursts with the metallicity: they are brighter, longer and have a slower decline at the lower metallicity of the SMC ([6]).

We also searched for BeSC using photometric criteria on 48 fields of the Galactic bulge within the OGLE-II catalog of stars. To determine if the selected stars were BeSC, we needed to inspect visually more than one hundred thousand light curves. This motivated us to implement a statistical criterion to select only the stars with light curves similar to those of the LMC, SMC and Galactic BeSC. A correlation between skewness and kurtosis was very useful to select the stars, guaranteeing the recovery of BeSC at the $70 \%$ level. This process conducted us to a catalog of about $29000 \mathrm{BeSC}$. We found that 198 of these stars exhibit a periodic behavior ([7]).

\footnotetext{
$\star$ bsabogal@uniandes.edu.co

$\star \star$ josegarc@uniandes.edu.co
} 
We also developed a search for BeSC within the ASAS catalog of variable stars ([8]). These Galactic stars are located mainly in the Galactic disk at different distances. For this reason, we could not use photometric criteria. Instead, we used the statistical criterion introduced by [7], using the ranges of skewness and kurtosis found in that work for BeSC in the I-band. Once a sample of stars was selected by this criterion, we applied morphological and photometric constraints and found 213 BeSC, showing outbursts and stochastic variability. We did not find stars with jumps in brightness in their light curves, suggesting that, for the detection range of ASAS telescopes, these stars do not exist in the Galaxy.

The advent of machine learning techniques to classify light curves of variable stars $([2,9])$ motivated us to implement a useful technique to select BeSC. We first propose and evaluate a set of six features extracted from the light curves. We used as training sample the following OGLE-III variable stars: Cepheids, RR Lyrae, $\delta$ Scuti, long-period variables, type II Cepheids, eclipsing binaries, a set of OGLE-III BeSC reported in the literature, and samples of BeSC reported in previous works. The best classifier to select BeSC was random forests ([4]). We used this classifier, and later color criteria, to search for BeSC within the OGLE-IV Gaia South Ecliptic Pole Field data. The result was a sample of 50 new BeSC, besides 16 BeSC previously reported by [10]. We also found stars with jumps of brightness that could belong to the LMC, due to the proximity of these fields to the center of that galaxy.

\section{Conclusions}

In this work we have shown the usefulness of combining photometric criteria with statistical criteria to select BeSC. The random forest method implemented with the features found in this work will be a very useful tool to select automatically BeSC from large databases.

Acknowledgments: Authors acknowledge support from Departamento de Física and Facultad de Ciencias, Universidad de los Andes, through CENIF program.

\section{References}

[1] Collins, G. W., in Physics of Be stars, Proc. IAU Coll. 92, ed. A. Slettebak \& T. P. Snow, Cambridge University Press (1987)

[2] Debosscher, J. Sarro, L. M., Aerts, C., Cuypers, J., Vandenbussche, B., Garrido, R., \& Solano, E., A\&A, 475, 1159 (2007)

[3] Mennickent, R. E., Pietrzyński, G., Gieren, W., \& Szewczyk, O., A\&A, 393, 887 (2002)

[4] Pérez-Ortiz, M.F., García-Varela, A., Sabogal, B., \& Quiroz, A., A\&A, submitted (2017)

[5] Rivinius, Th., Carciofi, A. C., \& Martayan, Ch., A\&ARv, 21, 69 (2013)

[6] Sabogal, B., Mennickent, R., Pietrzyński, G., \& Gieren, W., MNRAS, 361, 1055 (2005)

[7] Sabogal, B., Mennickent, R., Pietrzyński, G., García, J.A, Gieren, W., \& Kolaczkowski, Z., A\&A, 478, 659 (2008)

[8] Sabogal, B., García-Varela, A., \& Mennickent, R., PASP, 126, 219 (2014)

[9] Sarro, L. M., Debosscher, J., López, M., \& Aerts, C., A\&A, 494, 739 (2009)

[10] Soszyński, I., Udalski, A., Poleski, R., et al., Acta Astron., 62, 219 (2012) 